\title{
Self-interaction-free density-functional theoretical study of the electronic structure of spherical and vertical quantum dots
}

\author{
T. F. Jiang, ${ }^{*}$ Xiao-Min Tong, and Shih-I Chu \\ Department of Chemistry, University of Kansas, and Kansas Center for Advanced Scientific Computing, Lawrence, Kansas 66045
}

(Received 13 July 2000; published 9 January 2001)

\begin{abstract}
We study the electronic structure and shell-filling effects of both spherical and vertical quantum dots by means of the density functional theory (DFT) with optimized effective potential (OEP) and self-interactioncorrection (SIC) recently developed. The OEP/SIC procedure allows the elimination of the spurious selfinteraction energy and the construction of accurate single-particle local potential with proper long-range Coulombic behavior. The OEP/SIC equations are discretized and solved accurately and efficiently by the generalized pseudospectral (GPS) method. The highest occupied orbital energy of $N$-electron quantum dots provides a direct measure of the electron affinity or chemical potential. We apply the OEP/SIC method to the study of the capacitive energy of $N$-electron spherical dots for $N$ up to 70 . The results show the shell and subshell structure pattern and the electron filling pattern follows closely the Hund's rule. We also consider the effect of including the vertical dimension in the quantum dot study. We perform a detailed study of the shell-filling effect and the angular and radial density distributions of vertical quantum dots. The calculated capacitive energy spectrum is in good agreement with the recent experimental results, providing physical insights regarding the origin of electron shells and the role of electron-electron interaction in quantum dots.
\end{abstract}

DOI: 10.1103/PhysRevB.63.045317

PACS number(s): 73.21.La, 73.23.Hk, 73.61.-r

\section{INTRODUCTION}

The recent advancement in semiconductor nanostructure technology has led to the fabrication of zero-dimensional structure called quantum dots. ${ }^{1,2}$ Essentially they are small islands of laterally confined quasi-two-dimensional electrons. The study of the electronic structure of these confined electrons is significant to both basic physics and applied technology. The confining potential of the order of a few hundreds meV can now be arranged experimentally. Manybody effects due to the electron-electron interactions show a broad range of electronic structures similar to those of real atoms. The number of electrons in a quantum dot $N$ can be controlled experimentally, allowing the study of various physical properties of the quantum dots. The dependence of the chemical potential $\mu$ on $N$ can be measured directly through single-electron spectroscopy. ${ }^{3}$ By varying the size of quantum dot and the number of electrons, far IR absorption, ${ }^{4,5}$ capacitance spectroscopy, ${ }^{6}$ and conductance measurements ${ }^{3}$ etc., can be used to determine the tunneling conductance and capacitance resulting from the competition of quantum confinements and Coulomb interactions.

The experimental study of the addition energy spectra (or lately called the capacitive energy spectra because the measurement was made through the conductance fluctuations) of vertical quantum dots has been recently performed by Tarucha et al. ${ }^{7}$ Their vertical dot is cylindrical disk $\mathrm{In}_{0.05} \mathrm{Ga}_{0.95} \mathrm{As}$ with diameter around $10^{2} \mathrm{~nm}$ that is about ten times the thickness. The experimental addition energy of the dots up to 22 electrons showed interesting shell-filling structure features with magic numbers $2,6,12, \ldots$, etc. For electron confined in such a small length scale, the quantum effects are certainly very important. There have been intensive studies of the many-electron states in quantum dots. Direct diagonalization of the Hamiltonian is limited to cases of a few electrons. ${ }^{8}$ The Hartree-Fock calculation works for more electrons, but it does not take into account the correlation among electrons properly. The density-functional theory (DFT) (Ref. 9) has been used in the past few years. Since the vertical quantum dots are very thin disks, the $2 \mathrm{D}$ circular dots (in $x y$ plane only) are often used to model such systems. ${ }^{10-12}$ However, the 2D models have recently been found to be inadequate for the description of Coulomb interaction under single-site Hubbard and Hartree-Fock approaches. ${ }^{13}$ Although the small thickness in the $z$ direction of the quantum dots constrains the electronic states in the first subband, there are still distributions of electron probability along the vertical direction. Due to such strong confinement in the $z$ direction, the Coulomb integral and the kinetic energy are sensitively dependent on the electron density distribution. Thus, the inclusion of vertical dimension in calculation to model the experimental situation would be more realistic and desirable. However, the investigations including the $z$ dependence are rather rare. In a recent study by Lee et al. ${ }^{14}$ they investigated the parabolic and nonparabolic confinements in the $x y$ plane for the three-dimensional quantum dots. The Kohn-Sham equation was solved in Cartesian coordinates by a finite difference method and a large number of grid points typically about 138000 were used. They found that the introduction of small anisotropic confinement potential spoils the magic-number shell structure. Since the experimental capacitive energy spectra ${ }^{7}$ clearly show the existence of the magic number shell structures, we shall focus our present study on the isotropic confinement case only.

Most of the recent theoretical studies of the shell-filling behavior have been limited to the few-electron quantum dot cases, with the number of electrons $(N)$ less than $20 .{ }^{10-12,14}$ The detailed exploration of the general shell-filling behavior for many-electron quantum dots has yet to be performed. Moreover, all the DFT studies of quantum dots so far ${ }^{10-12,14}$ used the local spin density approximation (LSDA) (Refs. 15 
and 16) or generalized gradient approximation (GGA) (Refs. 17-19) energy functionals. Due to the existence of the spurious self-interaction energy, the use of either LSDA or GGA explicit energy functionals leads to exchange(x)correlation(c) potentials that do not have the correct longrange behavior. ${ }^{15}$ As such, although the total energies of the ground states predicted by these DFT methods are rather accurate, the ionization potentials obtained from the highest occupied Kohn-Sham orbitals are typically 30-50\% too small. Some of such problems can be circumvented by means of the DFT with optimized effective potential (OEP) and self-interaction-correction (SIC) recently developed. ${ }^{20}$ The OEP/SIC procedure allows the elimination of the selfinteraction energy and the construction of single-particle local potential with proper short- and long-range behavior. The predicted ionization potentials obtained from the highest occupied orbitals agree with the experimental data well within a few percent across the periodic table $(Z=2-106) .{ }^{21}$ The steady-state OEP/SIC procedure has also recently been extended to the time domain and applied to the study of multiphoton and high-order nonlinear optical processes of atoms ${ }^{22}$ and molecules ${ }^{23,24}$ with very encouraging results.

The motivations of this paper are twofold. First, we extend the steady-state OEP/SIC procedure to the study of the electronic structure and shell-filling behavior of manyelectron 3D spherical quantum dots for $N=2$ to 70 . The OEP/SIC equations are solved by means of the generalized pseudospectral (GPS) method ${ }^{25,26}$ recently developed, allowing optimal nonuniform spatial grid discretization. It has been demonstrated in several recent studies of bound and resonance states that high-precision eigenvalues and eigenfunctions can be achieved by this method with the use of only a modest number of spatial grid points. ${ }^{25,26}$ Second, we include the vertical degree of freedom and explore the shellfilling effects and the angular and radial density distributions of vertical quantum dots in details. The predicted addition or capacitive energy spectrum is found to be in good agreement with the recent experimental results. ${ }^{7}$

The paper is organized as follows. In Sec. II, we outline the DFT/OEP-SIC procedure for the problems of quantum dots. In Sec. III, we present the results of the study of 3D spherical dots. The study of the vertical dots is presented in Sec. IV, including a direct comparison of the calculated results with the experimental data. This is followed by a conclusion in Sec. V.

\section{THEORETICAL METHOD}

The Hamiltonian for a quantum dot, in the effective atomic units, can be written as follows:

$$
H=\sum_{i=1}^{N} h\left(\mathbf{r}_{i}\right)+\sum_{i<j}^{N} \frac{1}{\left|\mathbf{r}_{i}-\mathbf{r}_{j}\right|},
$$

where

$$
h\left(\mathbf{r}_{i}\right)=-\frac{\nabla_{i}^{2}}{2}+\frac{1}{2} \omega_{\perp}^{2}\left(x_{i}^{2}+y_{i}^{2}\right)+\frac{1}{2} \omega_{z}^{2} z_{i}^{2} .
$$

Here we adopt an anisotropic parabolic potential with the cylindrical symmetry of the characteristic frequencies $\omega_{\perp}$ and $\omega_{z}$. The spherical dot is the special case of $\omega_{\perp}=\omega_{z}$. For the semiconductor material $\mathrm{In}_{0.05} \mathrm{Ga}_{0.95} \mathrm{As}$ used in the experiment, ${ }^{7}$ the effective electron mass $m^{*}=0.0648 m_{e}$ and dielectric constant $\epsilon=12.96$ are obtained by the Vegard's law from those parameters of InAs and GaAs, ${ }^{29}$ where $m_{e}$ is the mass of a free electron. We use the length unit in effective Bohr radius, $\epsilon \hbar^{2} / m^{*} e^{2}=10.58 \mathrm{~nm}$ and energy in unit of effective Hartree, $m^{*} e^{4} / \epsilon^{2} \hbar^{2}=10.50 \mathrm{meV}$.

In DFT, the many-electron system is solved by the KohnSham (KS) equation: ${ }^{9}$

$$
\begin{aligned}
\hat{H}_{\mathrm{KS}} \psi_{i \sigma}(\mathbf{r}) & =\left[-\frac{1}{2} \nabla^{2}+v_{\mathrm{eff}, \sigma}(\mathbf{r})\right] \psi_{i \sigma}(\mathbf{r}) \\
& =\varepsilon_{i \sigma} \psi_{i \sigma}(\mathbf{r}), \quad\left(i=1,2, \ldots, N_{\sigma}\right),
\end{aligned}
$$

where $v_{\text {eff, } \sigma}(\mathbf{r})$ is the effective one-electron potential and $\sigma$ is the spin index.

In Eq. (3), the effective potential is written as

$$
v_{\mathrm{eff}, \sigma}(\mathbf{r})=v_{\mathrm{ext}}(\mathbf{r})+\int \frac{\rho\left(\mathbf{r}^{\prime}\right)}{\left|\mathbf{r}-\mathbf{r}^{\prime}\right|} d^{3} \mathbf{r}^{\prime}+v_{\mathrm{xc}}(\mathbf{r}),
$$

where $v_{\text {ext }}$ is the external potential and $v_{x c}$ is the exchange $(x)$-correlation(c) potential. For the case of quantum dots considered, the external potential is

$$
v_{\text {ext }}(\mathbf{r})=\frac{1}{2} \omega_{\perp}^{2}\left(x^{2}+y^{2}\right)+\frac{1}{2} \omega_{z}^{2} z^{2} .
$$

The total electron density is determined by

$$
\begin{aligned}
\rho(\mathbf{r}) & =\sum_{\sigma} \sum_{i=1}^{N_{\sigma}}\left|\psi_{i \sigma}(\mathbf{r})\right|^{2} \\
& =\sum_{i=1}^{N_{\uparrow}}\left|\psi_{i \uparrow}(\mathbf{r})\right|^{2}+\sum_{i=1}^{N_{\downarrow}}\left|\psi_{i \downarrow}(\mathbf{r})\right|^{2} \\
& =\rho_{\uparrow}(\mathbf{r})+\rho_{\downarrow}(\mathbf{r}),
\end{aligned}
$$

and the total energy $E[N]$ for the $N$-electron ground state is given by

$$
\begin{aligned}
E[N] & =E\left[\rho_{\uparrow}, \rho_{\downarrow}\right] \\
& =T_{s}[\rho]+J[\rho]+E_{\mathrm{xc}}\left[\rho_{\uparrow}, \boldsymbol{\rho}_{\downarrow}\right]+\int v_{\mathrm{ext}}(\mathbf{r}) \rho(\mathbf{r}) d^{3} \mathbf{r} .
\end{aligned}
$$

Here $T_{s}$ is the kinetic energy functional of noninteracting electrons,

$$
T_{s}=\sum_{\sigma} \sum_{i=1}^{N_{\sigma}}\left\langle\psi_{i \sigma}\left|-\frac{1}{2} \nabla^{2}\right| \psi_{i \sigma}\right\rangle,
$$

$J[\rho]$ is the classical electron-electron repulsive energy,

$$
J[\rho]=\frac{1}{2} \iint \frac{\rho(\mathbf{r}) \rho\left(\mathbf{r}^{\prime}\right)}{\left|\mathbf{r}-\mathbf{r}^{\prime}\right|} d^{3} \mathbf{r} d^{3} \mathbf{r}^{\prime},
$$


and $E_{\mathrm{xc}}\left[\rho_{\uparrow}, \rho_{\downarrow}\right]$ is the exchange-correlation energy. The xc potential is obtained by

$$
v_{\mathrm{xc}}(\mathbf{r})=\frac{\delta E_{\mathrm{xc}}\left[\boldsymbol{\rho}_{\uparrow}, \rho_{\downarrow}\right]}{\delta \rho_{\sigma}(\mathbf{r})} .
$$

Since the experimental addition energy spectrum was measured from the conductance fluctuations, the addition energy $\Delta A(N)$ is equal to the capacitive energy ${ }^{10}$ and is defined as

$$
\Delta A(N)=\mu(N+1)-\mu(N),
$$

where $\mu(N)=E(N)-E(N-1)$ is the chemical potential and $E(N)$ is the total energy of the $N$-electron system. The chemical potential is the energy required to add one electron to the system with $(N-1)$ electrons.

In this paper, we shall extend the OEP formalism with explicit SIC (Ref. 20) to the study of the electron structure of quantum dots. The OEP/SIC procedure ${ }^{20}$ is based on the extension of the semianalytic approach of Krieger-Li-Iafrate (KLI) (Ref. 27) for the solution of the OEP equations and the use of an explicit SIC form. ${ }^{28}$ As discussed in the introduction section, both the LSDA and GGA energy functionals commonly used in KS-DFT calculations contain spurious self-interaction contributions. The OEP-SIC formalism allows the elimination of the spurious self-interaction energy and the construction of more accurate effective potential with proper long-range Coulombic interaction. In the OEP formalism, one solves a set of one-electron equations, similar to the KS equations in Eq. (3),

$$
\begin{gathered}
\hat{H}_{\mathrm{OEP}} \psi_{i \sigma}(\mathbf{r})=\left[-\frac{1}{2} \nabla^{2}+V_{\sigma}^{\mathrm{OEP}}(\mathbf{r})\right] \psi_{i \sigma}(\mathbf{r})=\varepsilon_{i \sigma} \psi_{i \sigma}(\mathbf{r}), \\
\left(i=1,2, \ldots, N_{\sigma}\right) .
\end{gathered}
$$

The optimized effective potential, $V_{\sigma}^{\mathrm{OEP}}(\mathbf{r})$, is obtained by the requirement that the spin-orbital $\left\{\psi_{i \sigma}(\mathbf{r})\right\}$ in Eq. (12) are those that minimize the total energy functional $E\left[\psi_{i \uparrow}, \psi_{j \downarrow}\right]$ :

$$
\frac{\delta E^{\mathrm{OEP}}\left[\psi_{i \uparrow}, \psi_{j \downarrow}\right]}{\delta V_{\sigma}^{\mathrm{OEP}}(\mathbf{r})}=0,
$$

where

$$
\begin{aligned}
E^{\mathrm{OEP}}\left[\psi_{i \uparrow}, \psi_{j \downarrow}\right]= & T_{s}\left[\psi_{i \uparrow}, \psi_{j \downarrow}\right]+J\left[\psi_{i \uparrow}, \psi_{j \downarrow}\right]+E_{\mathrm{xc}}\left[\psi_{i \uparrow}, \psi_{j \downarrow}\right] \\
& +\int v_{\mathrm{ext}}(\mathbf{r}) \rho(\mathbf{r}) d^{3} \mathbf{r} .
\end{aligned}
$$

We shall adopt the following total energy functional with explicit (SIC) form $^{29}$

$$
\begin{aligned}
E_{\mathrm{SIC}}^{\mathrm{OEP}}\left[\psi_{i \uparrow}, \psi_{j \downarrow}\right]= & E^{\mathrm{OEP}}\left[\psi_{i \uparrow}, \psi_{j \downarrow}\right]-\sum_{\sigma} \sum_{i}\left\{J\left[\rho_{i \sigma}\right]\right. \\
& \left.+E_{\mathrm{xc}}\left[\rho_{i \sigma}, 0\right]\right\},
\end{aligned}
$$

where $E^{\mathrm{OEP}}\left[\psi_{i \uparrow}, \psi_{j \downarrow}\right]$ is given in Eq. (14). Following the KLI procedure, ${ }^{27}$ one arrives at

$$
\begin{aligned}
v_{\mathrm{eff}, \sigma}^{\mathrm{OEP}}(\mathbf{r})= & v_{\mathrm{ext}}(\mathbf{r})+\int \frac{\rho\left(\mathbf{r}^{\prime}\right)}{\left|\mathbf{r}-\mathbf{r}^{\prime}\right|} d^{3} \mathbf{r}^{\prime}+\frac{\delta E_{\mathrm{xc}}\left[\boldsymbol{\rho}_{\uparrow}, \boldsymbol{\rho}_{\downarrow}\right]}{\delta \rho_{\sigma}(\mathbf{r})} \\
& +V_{\mathrm{SIC}, \sigma}(\mathbf{r}),
\end{aligned}
$$

where

$$
\begin{gathered}
V_{\mathrm{SIC}, \sigma}(\mathbf{r})=\sum_{i} \frac{\rho_{i \sigma}(\mathbf{r})}{\rho_{\sigma}(\mathbf{r})}\left\{v_{i \sigma}(\mathbf{r})+\left[\bar{V}_{\mathrm{SIC}, \sigma}^{i}-\bar{v}_{i \sigma}\right]\right\}, \\
v_{i \sigma}(\mathbf{r})=-\int \frac{\rho_{i \sigma}\left(\mathbf{r}^{\prime}\right)}{\left|\mathbf{r}-\mathbf{r}^{\prime}\right|} d^{3} \mathbf{r}^{\prime}-\frac{\delta E_{\mathrm{xc}}\left[\rho_{i \sigma}, 0\right]}{\delta \rho_{i \sigma}(\mathbf{r})}
\end{gathered}
$$

and

$$
\begin{aligned}
\bar{V}_{\mathrm{SIC}, \sigma}^{i} & =\left\langle\phi_{i \sigma}\left|V_{\mathrm{SIC}, \sigma}(\mathbf{r})\right| \phi_{i \sigma}\right\rangle, \\
\bar{v}_{i \sigma} & =\left\langle\phi_{i \sigma}\left|v_{i \sigma}(\mathbf{r})\right| \phi_{i \sigma}\right\rangle .
\end{aligned}
$$

The OEP/KLI-SIC effective potential gives rise to correct behavior in both short range and long range. ${ }^{20}$ Moreover, the electronic energy of the highest occupied orbital provides an excellent approximation to the ionization potential throughout the Periodic Table. ${ }^{21}$ As will be shown later, in the OEP/ KLI-SIC scheme, we can calculate the chemical potentials directly from the highest occupied electron orbital energies. For both KS and OEP/KLI-SIC calculations, the equations are to be solved self-consistently. In the following, we outline the explicit procedure for the solution of the KS equation using the LSDA energy functional. Extension to other energy functionals or OEP/KLI-SIC is straightforward.

In solving the KS equation (3), we expand the electron orbitals in spherical harmonics:

$$
\psi_{i \sigma}(\mathbf{r})=\sum_{l} \frac{\phi_{i \sigma, l}(r)}{r} Y_{l m}(\Omega),
$$

where the magnetic quantum number $m$ is a good quantum number for each orbital. With the expansion, the total electron density is given by

$$
\begin{aligned}
\rho(\mathbf{r}) & =\sum_{i \sigma} n_{i \sigma}\left|\psi_{i \sigma}(\mathbf{r})\right|^{2} \\
& =\sum_{i \sigma} n_{i \sigma} \sum_{k, l} \frac{\phi_{i \sigma, k}^{*}(r) \phi_{i \sigma, l}(r)}{r^{2}} Y_{k m}^{*}(\Omega) Y_{l m}(\Omega),
\end{aligned}
$$

where $n_{i \sigma}$ is the number of electrons in the $i \sigma$ orbital. By using the integral of the product of three spherical harmonics,${ }^{30}$ the total electron density can be rewritten as

$$
\rho(\mathbf{r})=\sum_{i \sigma} n_{i \sigma} \sum_{j} \rho_{i \sigma, j}(r) Y_{j 0}(\Omega),
$$

with 


$$
\begin{aligned}
\rho_{i \sigma, j}(r)= & (-1)^{m} \sum_{k, l} \frac{\phi_{i \sigma, k}^{*}(r) \phi_{i \sigma, l}(r)}{r^{2}} \\
& \times \sqrt{\frac{(2 j+1)(2 k+1)(2 l+1)}{4 \pi}} \\
& \times\left(\begin{array}{lll}
k & l & j \\
0 & 0 & 0
\end{array}\right)\left(\begin{array}{ccc}
k & l & j \\
-m & m & 0
\end{array}\right),
\end{aligned}
$$

where

$$
\left(\begin{array}{ccc}
l_{1} & l_{2} & l_{3} \\
m_{1} & m_{2} & m_{3}
\end{array}\right)
$$

is the Wigner 3-j symbol. ${ }^{30}$

The LSDA exchange potential used here is 9

$$
V_{x, \sigma}^{\mathrm{LSDA}}(\mathbf{r})=-\left[\frac{6}{\pi} \rho_{\sigma}(\mathbf{r})\right]^{1 / 3}
$$

and the LSDA correlation potential has the VWN form. ${ }^{16} \mathrm{We}$ expand the exchange-correlation potentials in terms of spherical harmonics

$$
V_{\mathrm{xc}, \sigma}^{\mathrm{LSDA}}(\mathbf{r})=\sum_{j} V_{\mathrm{xc}, j}(r) Y_{j 0}(\Omega) .
$$

The expansion coefficient functions are calculated through

$$
V_{\mathrm{xc}, j}(r)=\int V_{\mathrm{xc}, \sigma}^{\mathrm{LSDA}}(\mathbf{r}) Y_{j 0}(\Omega) d \Omega,
$$

with the Gauss-Legendre quadrature, the azimuthal angle $\left\{\theta_{i}\right\}$ are generated by the grids from 0 to $\pi$ with $\left\{\cos \theta_{i}\right\}$ the zeros of Legendre polynomial $P_{L+1}\left(\cos \theta_{i}\right)$ and $\left\{\omega_{i}\right\}$ are the corresponding weights, ${ }^{31}$

$$
V_{\mathrm{xc}, j}(r)=\sum_{k=1}^{L+1} V_{\mathrm{xc}, \sigma}^{\mathrm{LSDA}}\left(r, \theta_{k}\right) P_{j}\left(\cos \theta_{k}\right) \omega_{k} \sqrt{\pi(2 j+1)} .
$$

The electron-electron interaction is especially important in this problem. Instead of calculating directly the integral

$$
V_{\mathrm{Coul}, i \sigma}(\mathbf{r})=\sum_{i \sigma} \int \frac{\rho_{i \sigma}\left(\mathbf{r}^{\prime}\right)}{\left|\mathbf{r}-\mathbf{r}^{\prime}\right|} d \mathbf{r}^{\prime}
$$

we perform the Coulomb integral through the solution of the Poisson equation:

$$
\nabla^{2} V_{\mathrm{Coul}, i \sigma}(\mathbf{r})=-4 \pi \rho_{i \sigma}(\mathbf{r}) .
$$

Since the Coulomb potential is also a functional of the electron density, we can expand it in the following partial wave form:

$$
V_{\mathrm{Coul}, i \sigma}(\mathbf{r})=\sum_{j} \frac{u_{i \sigma, j}(r)}{r} Y_{j 0}(\Omega) .
$$

Then $u_{i \sigma, j}(r)$ satisfies

$$
\left[\frac{d^{2}}{d r^{2}}-\frac{j(j+1)}{r^{2}}\right] u_{i \sigma, j}(r)=-4 \pi r \rho_{i \sigma, j}(r)
$$

with the boundary condition

$$
u_{i \sigma, j}(R)=\frac{4 \pi}{2 j+1} \frac{1}{R^{j}} \int_{0}^{R} \rho_{i \sigma, j}\left(r^{\prime}\right)\left(r^{\prime}\right)^{j+2} d r^{\prime}
$$

The Poisson equation is solved to machine accuracy by means of the generalized pseudospectral (GPS) method. ${ }^{25,26}$ For the radial coordinate $r$, we first map the range $[0, \infty]$ or $[0, R]$ into $[1,-1]$ by using

$$
r=r(x)=L \frac{1+x}{1-x+\alpha},
$$

where $\alpha=2 L / R$ and $L$ is the mapping parameter. The collocation points $\left\{x_{k}\right\}$ and the corresponding weights are determined through the Gauss-Lobatto-Legendre quadrature. ${ }^{26}$

With the above partial wave decompositions, the KohnSham equation reduces to the following coupled-channel form:

$$
\begin{aligned}
{\left[-\frac{1}{2}\right.} & \left.\frac{d^{2}}{d r^{2}}+\frac{l(l+1)}{2 r^{2}}+\frac{1}{2} \omega_{\perp}^{2} r^{2}\right] \phi_{i \sigma, l}(r) \delta_{l^{\prime} l} \\
& +\sum_{l} \frac{1}{2}\left(\omega_{z}^{2}-\omega_{\perp}^{2}\right) r^{2} A_{l}^{l^{\prime} m} \phi_{i \sigma, l}(r) \\
& +\sum_{l} \sum_{k} B_{k l}^{l^{\prime} m} V_{k}(r) \phi_{i \sigma, l}(r) \\
= & \epsilon_{i \sigma} \phi_{i \sigma, l}(r) \delta_{l^{\prime} l},
\end{aligned}
$$

where

$$
A_{l}^{l^{\prime} m}=\int Y_{l^{\prime} m}^{*}(\Omega) \cos ^{2} \theta Y_{l m}(\Omega) d \Omega,
$$

and

$$
B_{k l}^{l^{\prime} m}=\int Y_{l^{\prime} m}^{*}(\Omega) Y_{k 0}(\Omega) Y_{l m}(\Omega) d \Omega
$$

In Eq. (35), $V_{k}(r)$ is the sum of partial components of radial functions in Eqs. (26) and (31). During the selfconsistent calculation of the Eq. (35), up to 80 radial grid points, 40 azimuthal angular grids, and 15 spherical harmonics are used to achieve the converged results for the case of largest number of electrons, $N \simeq 70$, considered. Since the parity and magnetic quantum number $m$ are good quantum numbers, and $l \geqslant m$, the maximal orbital angular momentum used is actually at least 30 . For smaller $N$, the required number of mesh points is correspondingly smaller. We will now present the results of the study of both the cases of spherical dots and vertical dots below, using both the KS and the OEP/ KLI-SIC frameworks. 
TABLE I. Comparison of the calculated total energies, orbital energies, and chemical potentials of a two-electron system in a spherical quantum dot corresponding to $\omega_{\perp}=\omega_{z}=0.50$. The energies are in effective atomic units.

\begin{tabular}{lccc}
\hline \hline & $E_{\text {total }}$ & $E_{\text {orbital }}$ & $\mu$ \\
\hline LSDA & 1.9653 & 1.4229 & 1.2153 \\
BLYP & 2.0165 & 1.4464 & 1.26659 \\
LSDA/KLI-SIC & 1.9937 & 1.2517 & 1.2437 \\
BLYP/KLI-SIC & 2.0034 & 1.2527 & 1.2534 \\
EXACT & 2.0006 & & 1.2506 \\
\hline \hline
\end{tabular}

\section{SPHERICAL DOTS}

In the spherical dots, $\omega_{\perp}=\omega_{z}$ in Eq. (5). The KS equation is spherical symmetric and can be conveniently solved in terms of spherical harmonics as described in the previous section. The spherical dots have been recently used in the theoretical studies of electrodynamic response ${ }^{32}$ and hyperRaman scattering. ${ }^{33}$

Table I shows the comparison of four different DFT calculations of the total energy, orbital energy, and addition energy, respectively, corresponding to the case of twoelectron quantum dots, with the 3D spherical harmonic oscillator as the confining potential $\left(\omega_{\perp}=\omega_{z}=0.50\right)$. The twoelectron system can be separated into the center-of-mass $(\mathrm{CM})$ and relative motions $(\mathrm{RM})$. Introducing the relative and center-of-mass coordinates and momenta $\mathbf{r}=\mathbf{r}_{1}-\mathbf{r}_{2}, \mathbf{R}$ $=\left(\mathbf{r}_{1}+\mathbf{r}_{2}\right) / 2, \mathbf{p}=\left(\mathbf{p}_{1}-\mathbf{p}_{2}\right) / 2, \mathbf{P}=\left(\mathbf{p}_{1}+\mathbf{p}_{2}\right)$, the Hamiltonian in Eqs. (1) and (2) can be separated into the following CM and RM Hamiltonians,

$$
H_{\mathrm{CM}}=\frac{\mathbf{P}^{2}}{2 M}+\frac{M}{2} \omega_{\perp}^{2}\left(X^{2}+Y^{2}\right)+\frac{M}{2} \omega_{z}^{2} Z^{2},
$$

and

$$
H_{\mathrm{RM}}=\frac{\mathbf{p}^{2}}{2 \mu}+\frac{\mu}{2} \omega_{\perp}^{2}\left(x^{2}+y^{2}\right)+\frac{\mu}{2} \omega_{z}^{2} z^{2}+\frac{1}{\epsilon r},
$$

where $M=2 m^{*}$ and $\mu=m^{*} / 2$. The CM part is just a threedimensional harmonic oscillator, and the relative motion contains two interacting electrons under a harmonic potential. The radial part of the relative motion was solved numerically exactly by means of the generalized pseudospectral method. $^{25,26}$ The four DFT calculations correspond to the use of LSDA and the GGA type BLYP (Refs. 17 and 18) energy functionals with and without OEP/KLI-SIC. Also shown for comparison is the exact results for the total and addition energies. Without the OEP/KLI-SIC, the LSDA total energy is smaller than the exact energy, while the BLYP total energy is larger than the exact value. Further, the highest occupied orbital energy obtained from both LSDA and BLYP energy functionals differ substantially from the addition energy. With the implementation action of the OEP/KLI-SIC procedure, the total energies from both LSDA and BLYP energy functional forms are significantly improved, with the BLYP/KLI-SIC result closest to the exact value. More importantly, the highest occupied orbital energies from both

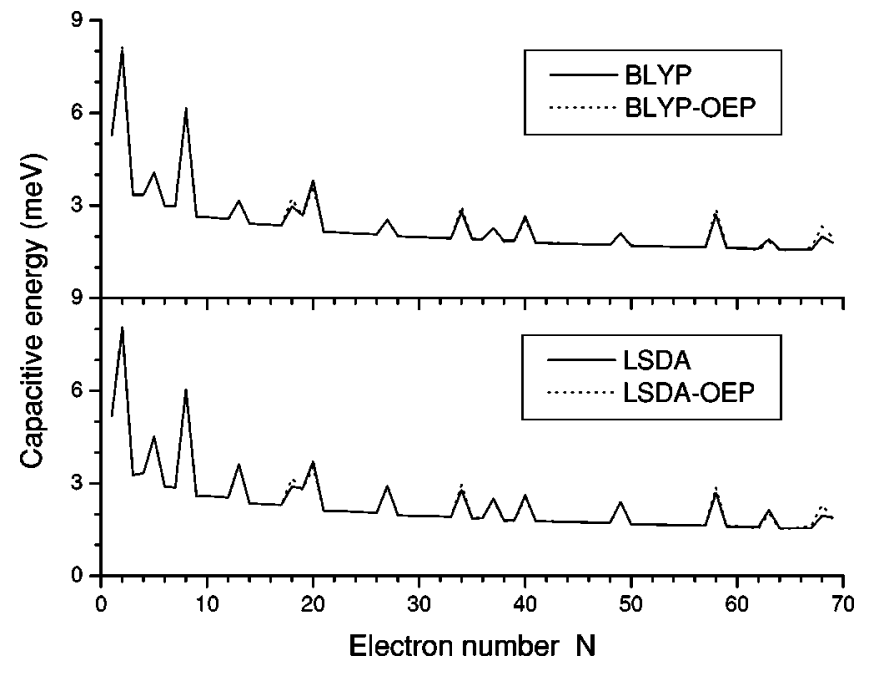

FIG. 1. The capacitive energies $[\Delta A(N)=\mu(N+1)-\mu(N)]$ of $N$-electron quantum dots confined by a spherical harmonic potential $\left(\omega_{\perp}=\omega_{z}=0.5\right)$, exhibiting the shell-filling structure. The upper figure shows the BLYP (solid line) and the BLYP/OEP-SIC (dashed line) results. The lower figure shows the LSDA (solid line) and LSDA/OEP-SIC (dashed line) results. The solid line corresponds to the results where the chemical potentials $\mu(N)$ are obtained by the total energy difference: $\mu(N)=E(N)-E(N-1)$. The dashed line shows the results where the chemical potentials $\mu(N)$ are obtained directly from the highest occupied orbital energies.

LSDA/KLI-SIC and BLYP/KLI-SIC calculations are close to the exact chemical potential. Physically this means that the highest occupied orbital energy of $N$-electron quantum dots can be used as a direct measure of the chemical potential $\mu(N)$. This is because the long-range potential in the OEP/KLI-SIC procedure has the proper Coulombic behavior and the electronic structure of quantum dots is more accurately described.

Figure 1 shows the capacitive energy, $\Delta A(N)$, as a function of the electron number $N$ calculated by BLYP and BLYP/KLI-SIC methods (upper figure), as well as by the LSDA and LSDA/KLI-SIC methods (lower figure), respectively. It exhibits the detailed shell and subshell electronic structure of many-electron spherical quantum dots. We note that although the numerical numbers of capacitive energies differ by a few percents, all the methods give rise to the same shell and subshell structure patterns.

To understand the origin of the electron shell-filling structure, we need to explore first the energy level orderings of individual electron orbital. In the absence of electronelectron interaction, the total energy of a 3D spherical harmonic oscillator is

$$
E_{n_{r} l m}=\left(2 n_{r}+l+\frac{3}{2}\right) \omega_{\perp}=\left(N+\frac{3}{2}\right) \omega_{\perp},
$$

where $n_{r}=0,1,2,3, \ldots$, and $N=2 n_{r}+l$. We use the designation $\left(n_{r}, l\right)$ to denote the energy level with the radial quantum numbers $n_{r}$ and the orbital angular $l$ in the above equation. For each $\left(n_{r}, l\right)$ level, there is $(2 l+1)$ degeneracy due to the magic sublevels, $\mathrm{m}=-l,-l+1, \ldots, l$. The energy orderings can be grouped as 
TABLE II. The total energy of two-electron vertical dot calculated by numerical diagonalization of the exact two-electron Hamiltonian and by LSDA for $\omega_{\perp}=0.5$. The energies, $\omega_{\perp}$ and $\omega_{z}$, are in effective atomic units. The error indicates the percentage deviation of the LSDA from the exact numerical diagonalization.

\begin{tabular}{lccc}
\hline \hline$\omega_{z}$ & Diagonalization & LSDA & Error\% \\
\hline 0.5 & 2.000 & 2.026 & 1.30 \\
1.0 & 2.553 & 2.581 & 1.10 \\
2.0 & 3.590 & 3.607 & 0.47 \\
3.0 & 4.605 & 4.612 & 0.15 \\
4.0 & 5.614 & 5.614 & 0.01 \\
\hline \hline
\end{tabular}

$\{(0,0)\},\{(0,1)\},\{(0,2),(1,0)\},\{(0,3),(1,1)\},\{(0,4),(1,2),(2,0)\}, \ldots$ where the energy levels in the same bracket are degenerate. In the presence of many-body electron-electron interaction, the degeneracy will be lifted. If the electron-electron effects are not enough to change the shell orderings, the number of electrons in the closed shell will be $2,8,20,40,70, \ldots$. These are the magic numbers of spherical dots. From our calculations, we found that when the electron-electron effects are taken into account, the electron orbital in a shell with larger angular momentum lies lower in energy. This is reminiscent of the Hund's rule. For example, the degeneracy of $\{(0,2),(1,0)\}$ is lifted into nondegenerate states $(0,2)$ and $(1,0)$ while the orbital $(0,2)$ with higher angular momentum has the lower energy than $(1,0)$.

Using this energy ordering, we are able to identify all the shell structures in Fig. 1. The most prominent shell structure occurs at the following magic numbers $N=2,8,20,40, \ldots$, etc. corresponding to the fully occupied shells of $\left\{(0,0)^{2}\right\},\left\{(0,1)^{6}\right\},\left\{(0,2)^{10},(1,0)^{2}\right\},\left\{(0,3)^{14},(1,1)^{6}\right\}, \ldots$, etc. where the superscript denotes the number of electrons in the filled orbital, taking into account the degeneracy due to magnetic sublevels. The smaller peaks in Fig. 1 can also be identified as those quantum dots with half-filled subshells. It is instructive to find that the Hund's rule is also applicable to the spherical quantum dot systems.

\section{VERTICAL DOTS}

To mimic the large ratio of planar diameter to the height of the vertical dots as used in the experiment, ${ }^{7}$ we choose $\omega_{z} \gg \omega_{\perp}$ in Eq. (5). Since the ground-state wave function spreading of a harmonic oscillator is inversely proportional to the square root of its natural frequency, the larger value of $\omega_{z}$ ensures that the electron probability distribution in the vertical direction is confined within a small distance. ${ }^{14}$

A two-electron system in a vertical dot can also be separated into relative and center-of-mass motions. The relative Hamiltonian can then be discretized and diagonalized to high accuracy by means of the generalized pseudospectral method. ${ }^{25,26}$ Table II shows the comparison of the LSDA total energy and the exact numerical results for the case of $\omega_{\perp}=0.5$ and $\omega_{z}$ from 0.5 to 4.0 . We see that for $\omega_{z}>3$, our calculation errors (LSDA column) for the two-electron system in a vertical dot are below 0.15\%. In Fig. 2 we show the capacitive energy of the two-electron vertical quantum dot vs

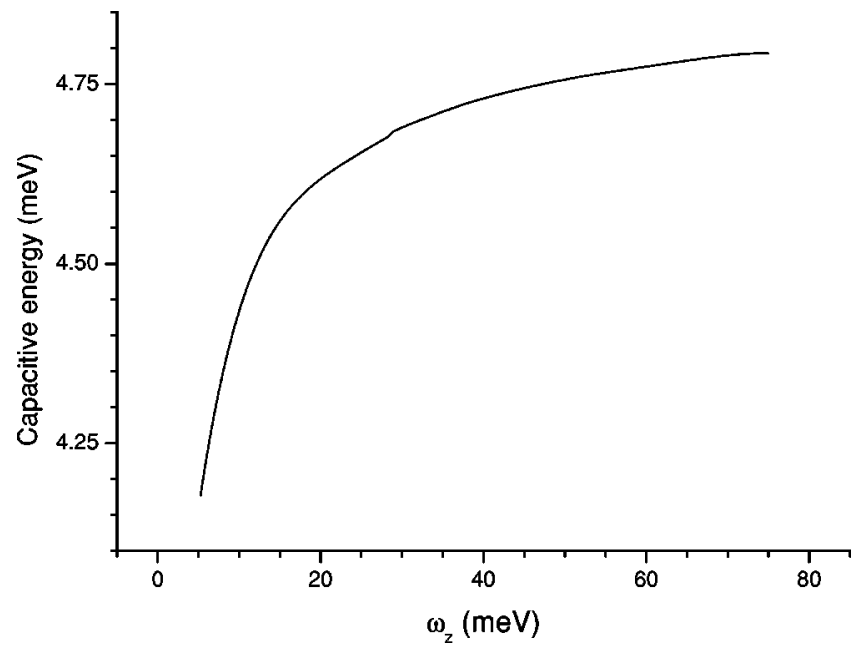

FIG. 2. The capacitive energy of two-electron system in a vertical quantum dot vs $\omega_{z}$. It is seen that the capacitive energy saturates for larger value of $\omega_{z}$. Here $\omega_{\perp}=3 \mathrm{meV}$ and one effective atomic unit corresponds to $10.505 \mathrm{meV}$.

the values of $\omega_{z}$. The capacitive energy saturates for larger value of $\omega_{z}$. We note that the computational effort goes up rapidly with increasing value of $\omega_{z}$ as more angular momentum is required to achieve convergence. The saturation of the capacitive energy for sufficiently large value of $\omega_{z}$ justifies that the use of $\omega_{z}=5$ (effective) a.u. $=52.5 \mathrm{meV}$ in our LSDA calculations below is physically meaningful.

In order to simulate the experimental conditions, ${ }^{7}$ we set $\omega_{\perp}=3.0 \mathrm{meV}$, and $\omega_{z}=52.5 \mathrm{meV}$ in Eq. (5). These parameters mean that the planar dimension ( $x y$ plane) is much larger than the vertical $(z)$ dimension. ${ }^{14}$ Figure 3 depicts the angular density distribution along $\theta$ for $N=1,6,12$, and 20 electrons that include both closed and open shell cases, where $\theta=\pi / 2$ is in the disk planar direction. The angular density $\rho(\theta)$ is defined as

$$
\int \rho(\theta) d \theta=1 .
$$

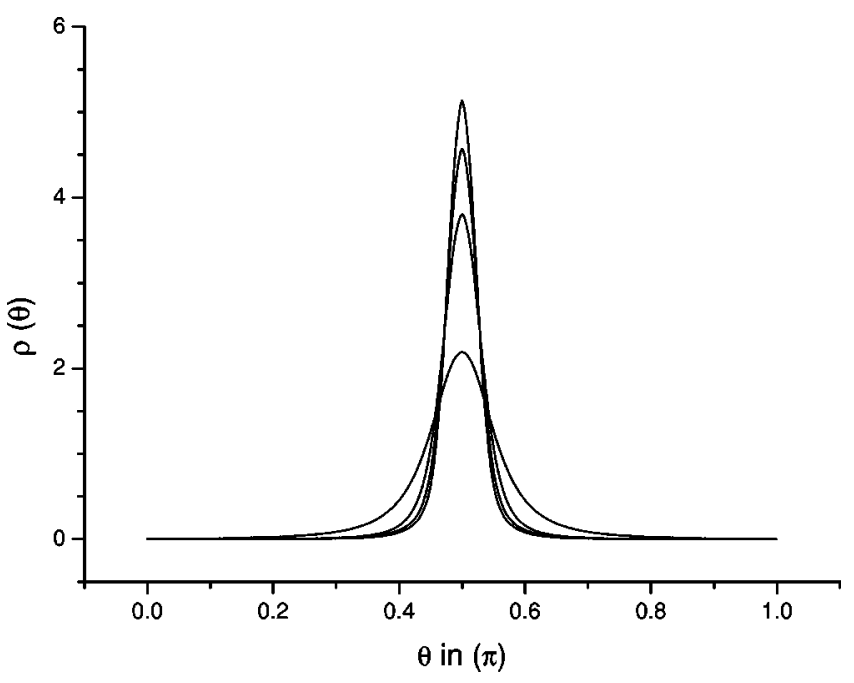

FIG. 3. Angular distribution of electron density in a vertical dot for $N=1,6,12$, and $20 . N$ starts at 1 from the innermost curve. 


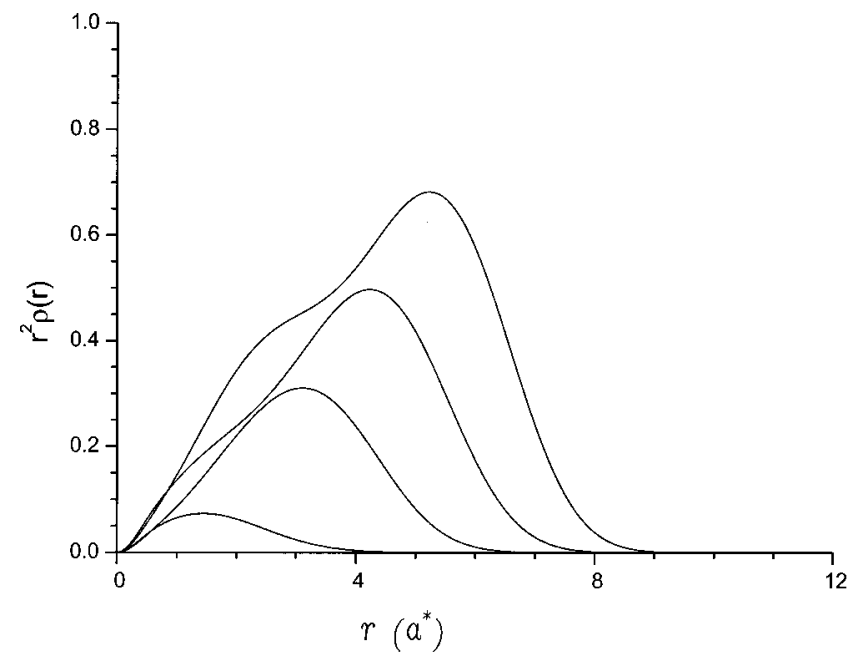

FIG. 4. Radial distribution of electron density in a vertical dot for $N=1,6,12$, and $20 . N$ starts at 1 from the innermost curve. Note that the peak position increases with $N . r$ is in unit of effective Bohr radius $a^{*}$.

The results show that the electrons are confined more sharply along the planar region as the number of electrons $N$ increases. This explains why several 2D planar disk model calculations ${ }^{10-12}$ showed roughly the shell structure correctly but the details might not be reliable for the $\theta$ distributions are neglected. As indicated in Ref. 13, the 2D model description of Coulomb interaction is quite different from that of the real system in the simple 2D model. Also we find that for systems with just a few electrons, the angular distribution is actually rather broad, indicating the $2 \mathrm{D}$ approximation would be inappropriate for such situations.

To have a more complete picture of many-electron density distribution in quantum dots, we show in Fig. 4 the corresponding radial electron density distributions. Here the radial density is defined as

$$
\int \rho(r) r^{2} d r=1
$$

It is instructive to note that the maximum of total electron probability for a given $N$-electron vertical dot occurs at certain special radius like that in atomic systems. And the peak position occurs at larger distance as the electron number $N$ increases. We also perform the calculation with $\omega_{\perp}$ $=3 \mathrm{meV}$ and $\omega_{z}=31.5 \mathrm{meV}$. Similar angular and radial distributions, and addition energy spectra were obtained. This is because the motion of electrons in the $z$ direction is mainly confined in the first subband, so the planar lateral confined potential determines the main properties of the electron states. Thus the results are nearly independent of the value of $\omega_{z}$.

Figure 5 shows the $N$-dependent capacitive energy spectra. In order to compare with the experimental results, ${ }^{7}$ we first calculate the vertical dots with $\omega_{\perp}=3 \mathrm{meV}$, the diameter $D=500 \mathrm{~nm}$ and $\omega_{z}=52.5 \mathrm{meV}$. The results show clearly not only the magic-number shell structures at $2,6,12,20, \ldots$, but also the correct magnitude of capacitive

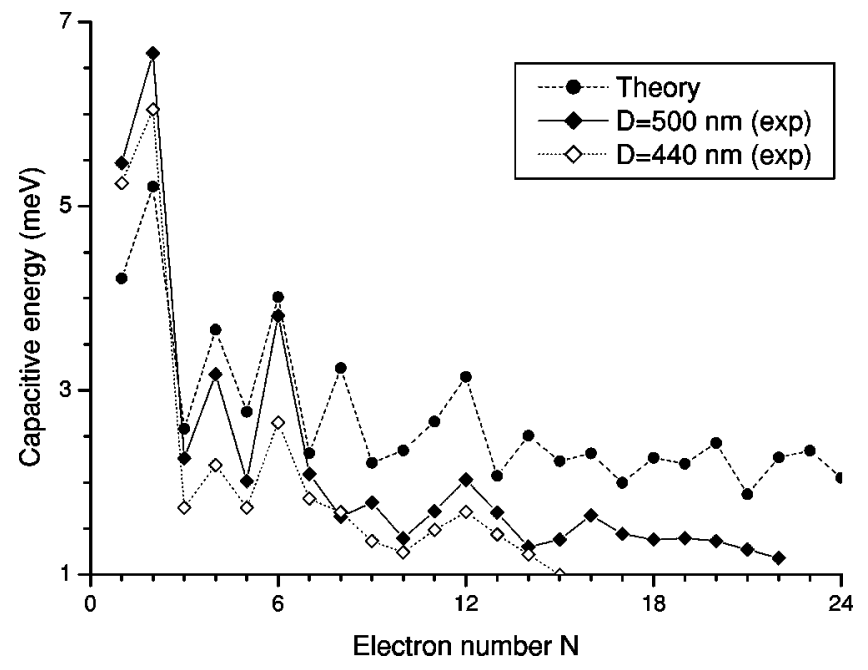

FIG. 5. The capacitive energy spectrum for vertical quantum dots with $\omega_{\perp}=3 \mathrm{meV}$ and $\omega_{z}=52.5 \mathrm{meV}$. The lower two curves are the experimental results (Ref. 7), and the top curve is the current theoretical prediction, which is independent of the value of $D$ used.

energy. There are also subpeaks at $N=4,8,14, \ldots$, etc. These subpeaks were not clear in Refs. 10 and 14, but were identified in both the experiment data ${ }^{12}$ and our present calculations. These subpeaks can be attributed to the Hund's rule for half-filled shells. However, when the electron number becomes larger, the electron-electron interactions weaken the shell structure arising from the binding planar parabolic confined potential. Within the same subshell, the electron filling follows the Hund's rule that the states of the same spin will fill first. Since the electronic states must be antisymmetric, electrons of the same spin tend to repel each other spatially, the Coulomb energy between them will then be lowered significantly. On the other hand, if two electrons have different spins, their spatial overlap can be larger and the total energy will be higher. We can see in the plot that the Hund's rule are actually followed.

We then performed a calculation with the same parameters $\omega_{\perp}$ and $\omega_{z}$ but the diameter $D$ is now $440 \mathrm{~nm}$ as used in the experiment. ${ }^{7}$ We found no noticeable difference in the capacitive energy spectra for the cases of $D=440 \mathrm{~nm}$ and $D=500 \mathrm{~nm}$. This is expected since the extent of the radial electron density distribution is much less than $D$ in these cases as shown Fig. 4. Thus, the cases of $D=440 \mathrm{~nm}$ and $D=500 \mathrm{~nm}$ are physically the same for the range of electron number $N$ we investigated. However, the experimental data ${ }^{7}$ for the two different $D$ cases showed notable difference. This suggests that the commonly used parabolic lateral confined potential such as that in Eq. (2) may need some refinement to include the dependence of diameters of quantum dots.

\section{CONCLUSION}

In this paper, we present a DFT with OEP and SIC for the detailed study of the electronic structure of parabolically confined quantum dots. The OEP/SIC procedure allows the elimination of the spurious self-interaction energy in the 
conventional DFT calculations using explicit LSDA or GGA energy functionals, providing the proper long-range effective potential and more accurate description of both ground and excited states. The electronic energy of the highest occupied Kohn-Sham orbital (with OEP/SIC) provides an accurate and direct measure of the electron affinity or chemical potential of the quantum dot systems. The OEP/SIC equations are solved accurately and efficiently by means of the the generalized pseudospectral method, allowing nonuniform spatial grid discretization and the use of only a modest number of grid points.

We first study the electronic structure of $N$-electron spherical quantum dots for $N$ up to 70 using both LSDA and BLYP energy functionals with or without the use of OEP/ SIC. The calculated capacitive energy spectra shows shell and subshell structures analogous to those in atomic systems. The electron filling pattern is found to follow closely the Hund's rule.

We also study the electronic structure and electron filling pattern of vertical quantum dots by means of DFT. We found that the commonly used 2D model is too crude to model the realistic quantum dots. We explore the angular and radial electron density distributions in $\mathrm{N}$-electron quantum dots. The density distribution of few-electron dots in the vertical direction is found to be broader than that in the manyelectron dots. Our calculated capacitive energy spectrum reproduces the shell and subshell features seen in the experiments. ${ }^{7}$ There is still some discrepancy in the predicted and experimental data, namely, the experimental capacitive energy depends upon the value of the diameter $D$ (500 or 440 $\mathrm{nm}$ ) used, while our calculations do not. This discrepancy indicates that future refinement of the confining potential must take into account the diameter $(D)$ dependence in order to achieve better agreement with the experimental observation.

Finally, due to the generalized Kohn theorem ${ }^{34}$ associated with the parabolically confined potentials, the far-infrared spectra can show only the motion of center-of-mass part for electrons in the quantum dots. The electron-electron interaction in the relative motion part is hard to detect. There are several proposed methods that can be used to measure the relative motion part such as the magnetization, heat capacity etc. $^{35}$ The accurate calculation of the capacitive spectrum presented in this paper can be used as another way to explore the relative motion of many-electron system in the parabolic quantum dots.

\section{ACKNOWLEDGMENTS}

This work was partially supported by NSF No. PHY9801889. We are grateful to Kansas Center for Advanced Scientific Computing (KCASC) for the support of Origin 2000 supercomputer time. T.F.J. acknowledges the supports from KCASC and National Science Council, Taiwan. We are grateful to $\mathrm{Xi} \mathrm{Chu} \mathrm{for} \mathrm{the} \mathrm{assistance} \mathrm{of} \mathrm{the} \mathrm{final} \mathrm{draft} \mathrm{of} \mathrm{this}$ paper.
*Permanent address: Institute of Physics, National Chiao Tung University, Hsinchu 30010, Taiwan.

${ }^{1}$ M. Kastner, Phys. Today 46, 24 (1993); R.C. Ashoori, Nature (London) 379, 413 (1996).

${ }^{2}$ L. Jacak, P. Hawrylak, and A. Wojs, Quantum Dots (SpringerVerlag, New York, 1989).

${ }^{3}$ R.C. Ashoori et al., Phys. Rev. Lett. 71, 613 (1993).

${ }^{4}$ A. Lorke, J.P. Kotthaus, and K. Ploog, Phys. Rev. Lett. 64, 2559 (1990).

${ }^{5}$ C. Sikorski and U. Merkt, Phys. Rev. Lett. 62, 2164 (1989).

${ }^{6}$ W. Hansen et al., Phys. Rev. Lett. 62, 2168 (1989).

${ }^{7}$ S. Tarucha, D.G. Austing, T. Honda, R.J. van der Hage, and L.P. Kouwenhoven, Phys. Rev. Lett. 77, 3613 (1996).

${ }^{8}$ P.A. Maksym and T. Chakraborty, Phys. Rev. Lett. 65, 108 (1990); P. Hawrylak and D. Pfannkuche, ibid. 70, 485 (1993); J.J. Palacios et al., Phys. Rev. B 50, 5760 (1994); S.R.E. Yang, A.H. MacDonald, and M.D. Johnson, Phys. Rev. Lett. 71, 3194 (1993).

${ }^{9}$ P. Hohengerg and W. Kohn, Phys. Rev. 136, B864 (1964); W. Kohn and L.J. Sham, ibid. 140, A1133 (1965).

${ }^{10}$ M. Macucci, K. Hess, and G.J. Iafrate, Phys. Rev. B 48, 17354 (1993); M. Macucci, K. Hess, and G.J. Iafrate, J. Appl. Phys. 77, 3267 (1995); G.J. Iafrate, K. Hess, J.B. Krieger, and M. Macucci, Phys. Rev. B 52, 10737 (1995); M. Macucci, K. Hess, and G.J. Iafrate, ibid. 55, 4879 (1997).

${ }^{11}$ M. Koskinen, M. Manninen, and S.M. Reimann, Phys. Rev. Lett. 79, 1389 (1997).

${ }^{12}$ K. Hirose and N.S. Wingreen, Phys. Rev. B 59, 4604 (1999).

${ }^{13}$ M. Rontani, F. Rossi, F. Manghi, and E. Molinari, Phys. Rev. B
59, 10165 (1999).

${ }^{14}$ I.H. Lee, V. Rao, R.M. Martin, and J.P. Leburton, Phys. Rev. B 57, 9035 (1998).

${ }^{15}$ R.G. Parr and W.T. Yang, Density-Functional Theory of Atoms and Molecules (Oxford University Press, New York, 1989).

${ }^{16}$ S.J. Vosko, L. Wilk, and M. Nusair, Can. J. Phys. 58, 1200 (1980).

${ }^{17}$ A.D. Becke, Phys. Rev. A 38, 3098 (1988).

${ }^{18}$ C. Lee, W. Yang, and R.G. Parr, Phys. Rev. B 37, 785 (1988).

${ }^{19}$ J.P. Perdew and Y. Wang, Phys. Rev. B 33, 8800 (1986).

${ }^{20}$ X.M. Tong and S.I. Chu, Phys. Rev. A 55, 3406 (1997).

${ }^{21}$ X.M. Tong and S.I. Chu, Phys. Rev. A 57, 855 (1998).

${ }^{22}$ X.M. Tong and S.I. Chu, Phys. Rev. A 57, 452 (1998); 58, R2656 (1998).

${ }^{23}$ X. Chu and S.I. Chu, Conf. Proc., International Conference on Multiphoton Processes (2000).

${ }^{24}$ X. Chu and S.I. Chu, Phys. Rev. A (to be published).

${ }^{25}$ G. Yao and S.I. Chu, Chem. Phys. Lett. 204, 381 (1993).

${ }^{26}$ J. Wang, S.I. Chu, and C. Laughlin, Phys. Rev. A 50, 3208 (1994).

${ }^{27}$ J.B. Krieger, Y. Li, and G.J. Iafrate, Phys. Rev. A 46, 5453 (1992); Y. Li, J.B. Krieger, and G.J. Iafrate, ibid. 47, 165 (1993).

${ }^{28}$ J.P. Perdew and A. Zunger, Phys. Rev. B 23, 5048 (1981).

${ }^{29}$ Semiconductors-Basic Data, edited by O. Madelung, 2nd ed. (Springer-Verlag, Berlin, 1996).

${ }^{30}$ A.R. Edmonds, Angular Momentum in Quantum Mechanics (Princeton, New Jersey, 1974).

${ }^{31}$ W.H. Press, E.A. Teukolsky, W.T. Vetterling, and B.P. Flannery, 
Numerical Recipes in Fortran, 2nd ed. (Cambridge, New York, 1992).

${ }^{32}$ Q.P. Li, K. Karrai, S.K. Yip, S. Das Sarma, and H.D. Drew, Phys. Rev. B 43, 5151 (1991).
${ }^{33}$ E.M. Proupin, J.L. Pena, and C.T. Giner, Semicond. Sci. Technol. 13, 871 (1998).

${ }^{34}$ W. Kohn, Phys. Rev. 123, 1242 (1961).

${ }^{35}$ P.A. Maksym and T. Chakraborty, Phys. Rev. B 45, 1947 (1992). 